marker for disease activity): 'easy-to-treat RA' and 'difficult-to-treat RA'.

Proinflammatory and anti-inflammatory cytokine levels changed after mirthful laughter in easy-to-treat and difficult-to-treat patients, compared with no change in healthy participants; the extent of immunomodulation was dependent on disease activity. Since this study found favorable effects of mirthful laughter on the immune response, the authors suggest that psychological support should be offered as an adjunct to treatment for patients with RA.

Rachel Murphy

Original article Matsuzaki T et al. (2006) Mirthful laughter differentially affects serum pro- and anti-inflammatory cytokine levels depending on the level of disease activity in patients with rheumatoid arthrits. Rheumatology 45: 182-186

\section{Pilot study of vardenafil for the treatment of Raynaud's disease}

A German pilot study has shown that the phosphodiesterase-type-5 (PDE5) inhibitor vardenafil substantially improves peripheral blood flow and clinical symptoms in patients with Raynaud's disease. The investigators tested vardenafil in these patients because PDE5 inhibitors are known to exert vasodilatory effects.

The patients-33 with secondary Raynaud's disease and 7 with primary Raynaud's disease - received $10 \mathrm{mg}$ vardenafil twice daily for 2 weeks. Peripheral blood flow in the index finger was measured at room temperature by laser Doppler flowmetry at baseline, and at $1 \mathrm{~h}$ and 2 weeks after initiation of therapy. Measurements were repeated during a cold-exposure test at $4^{\circ} \mathrm{C}$. Patients completed a questionnaire that quantified the severity of their symptoms (the Raynaud's condition score).

Improvement in peripheral blood flow was observed in $70 \%$ of patients and improvement in clinical symptoms was reported by $68 \%$ of patients. During the cold-exposure test, vardenafil markedly increased blood flow at $1 \mathrm{~h}$ and at 2 weeks. There were no severe adverse effects of vardenafil, and the type and incidence of minor adverse events were similar to those seen in other studies of PDE5 inhibitors.

PDE5 inhibitors have a different mechanism of action from the established Raynaud's disease treatment of calcium-channel blockers and, therefore, combination therapy could show an enhanced therapeutic effect.
A placebo-controlled comparison and/or combination trial with PDE5 inhibitors and established medications is warranted.

Rachel Murphy

Original article Caglayan E et al. (2006) Phosphodiesterase type 5 inhbition is a novel therapeutic option in Raynaud disease. Arch Intern Med 166: 231-233

\section{Vaccination against influenza is safe in patients with rheumatoid arthritis}

Infection in rheumatoid arthritis $(\mathrm{RA})$ is associated with considerable morbidity and mortality, possibly because of disease-related immune dysfunction and treatment with immunosuppressive drugs. The safety of vaccination in patients with RA is disputed; disease flares following vaccination have been reported, and the immunogenicity of vaccines in immunocompromised individuals and the effect of disease-modifying antirheumatic drugs (DMARDs) have yet to be determined. Researchers in Israel investigated the safety and immunogenicity of influenza vaccine in 82 consecutive patients with RA, compared with 30 healthy controls. Each dose of vaccine contained $15 \mu \mathrm{g}$ hemagglutinin of each of $\mathrm{B} / \mathrm{Hong}$ Kong/330/2001, A/Panama/2007/99 and A/New Caledonian/20/99 subtypes. All RA patients were being treated with at least one DMARD.

Vaccination had no effect on RA disease activity. By 6 weeks after vaccination, both the RA group and the controls showed a good serum antibody response to the vaccine, although the response to the Hong Kong component was significantly higher in the control group $(P=0.004)$. The percentage of responders to the Hong Kong component was also significantly higher in the control group than in the RA group ( $87 \%$ versus $67 \%, P=0.05)$; responses to the other components were not significantly different. Prednisone and DMARDs had no effect on response to the vaccine. There were 12 reports of minor adverse effects $(7 / 82$ in the RA group and $5 / 30$ in the control group); 10 of these were upper-respiratory-tract infections.

The authors conclude that the vaccine is safe and produces a good humoral response in individuals with RA.

Jim Casey

Original article Fomin I et al. (2006) Vaccination against

influenza in rheumatoid arthritis: the effect of disease modifying drugs, including TNFa blockers. Ann Rheum Dis 65: 191-194 